\title{
Central diffractive resonance production at the LHC
}

\author{
Roberto Fiore $^{1, a}$, Laszlo Jenkovszky ${ }^{2, b}$, and Rainer Schicker ${ }^{3, c}$ \\ ${ }^{1}$ INFN and Dipartimento di Physica, Universita della Calabria, I-87036 Arcavacata di Rende, Cosenza, ITALY \\ ${ }^{2}$ Bogolyubov ITP, National Academy of Sciences of Ukraine, $03680 \mathrm{Kiev}$, UKRAINE \\ ${ }^{3}$ Physikalisches Institut, Im Neuenheimer Feld 226, Heidelberg University, 69120 Heidelberg, GERMANY
}

\begin{abstract}
Central production of resonances resulting from the scattering of Pomerons in the central rapidity region of proton-proton scattering is studied. Estimates for relevant cross sections are presented.
\end{abstract}

\section{Introduction}

Inelastic diffraction in the triple Regge limit was studied in quite a number of papers, see e.g. [1, 2]. With the advent of the LHC, the interest shifted to the low missing-mass region, below the asymptotic triple Regge region. Progress in theory became possible due to a dual-Regge approach, successfully applied, in the low missing mass region [3] to single- (SD) and double (DD) diffraction dissociation, diagrams 2 and 3 of Fig. 1. By the present contribution we make one more step by applying the above dual-Regge technique to central diffractive production of resonances at the LHC [4].

The total cross section is a sum of the integrated elastic, $\sigma_{e l}(s)$ and inelastic cross sections $\sigma_{\text {inel }}(s)$, the latter being a sum of single, double and central diffractive cross sections, plus the non-diffractive cross section. The proton-proton total cross section to a good approximation is a sum of a Pomeron and $f$ Reggeon contribution, symbolically, $\sigma_{t}=P+f$. A number of equally good fits to the total cross section exist, see e.g. [5], most of them being compatible with the negligible contribution of the secondary Reggions (here, that of the $f$ Reggeon) to total cross section at the LHC, $\sqrt{s} \geq 7 \mathrm{TeV}$.

We consider:

SD $\quad p p \longrightarrow X p \quad$ Single Diffraction Dissociation (or Single Diffraction),

or $\quad p p \longrightarrow p Y \quad$ Single Diffraction Dissociation (symmetric),

DD $\quad p p \longrightarrow X Y$ Double Diffraction Dissociation (Double Diffraction),

CED $p p \longrightarrow p X p \quad$ Central (Exclusive) Diffraction (or DPE) (or Double Pomeron Exchange). The "symmetric" $p p \longrightarrow X p$ and $p p \longrightarrow p Y$ cross sections, for simplicity, are assumed to be equal.

For large rapidity gaps, $\Delta y \geq 3$, or, equivalently, when Pomeron exchange dominates, in the triple Pomeron limit, the above cross sections can be written as product of the Pomeron flux (first factor in brackets) times the Pomeron-proton total cross section:

\footnotetext{
a e-mail: fiore@fis.unical.it

be-mail: jenk@bitp.kiev.ua; L.J. gratefully acknowledges an EMMI visiting Professorship at the University of Heidelberg for completion of this work. He is grateful to the organizers of this meeting for their hospitality and support. His work was supported also by DOMUS, Hungarian Academy of Sciences

ce-mail: schicker@physi.uni-heidelberg,de
} 


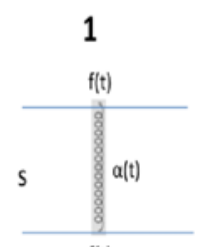

$\mathrm{f}(\mathrm{t})$
2
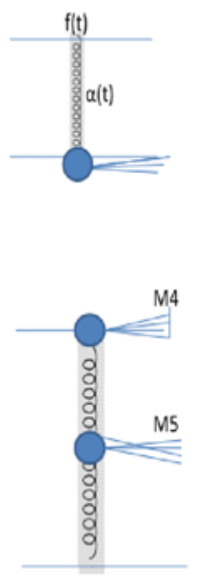

5
3
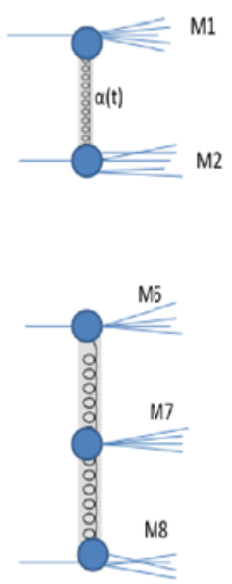

6

Figure 1. Regge-pole diagrams for elastic, single-, double- and central diffraction.

$$
\begin{aligned}
\frac{d \sigma_{S D}}{d t \Delta y} & =\left[\frac{\beta^{2}(t)}{16 \pi} e^{2[\alpha(t)-1] \Delta y}\right] \times\left[k \beta^{2}(0)\left(\frac{s^{\prime}}{s_{0}}\right)^{\epsilon}\right], \\
\frac{d \sigma_{D D}}{d t \Delta y y_{0}} & =\left[k \frac{\beta^{2}(t)}{16 \pi} e^{2[\alpha(t)-1] \Delta y}\right] \times\left[k \beta^{2}(0)\left(\frac{s^{\prime}}{s_{0}}\right)^{\epsilon}\right], \\
\frac{d \sigma_{D P E}}{d t_{1} d t_{2} \Delta y d y_{c}} & =\left[\prod_{i}\left(\frac{\beta^{2}\left(t_{i}\right)}{16 \pi} e^{2\left[\alpha\left(t_{i}\right)-1\right] \Delta y_{i}}\right)\right] \times k\left[k \beta^{2}(0)\left(\frac{s^{\prime}}{s_{0}}\right)^{\epsilon}\right],
\end{aligned}
$$

where $t$ is the square of the four-momentum transfer at the proton vertex and $\Delta y$ is the rapidity gap width. The subscript $i=1,2$ enumerates Pomerons in DPE; $\Delta y=\Delta y_{1}+\Delta y_{2}$ is the sum of two rapiditygap width in the same event, and $c_{i}$ is the center in $\eta$ of the centrally-produced hadronic system. Since our units below are in $\mathrm{GeV}$, we use $1 \mathrm{GeV}^{-2}=0.389 \mathrm{mb}$ in the final cross sections. We use the relations $\alpha(t)=1.104+0.25 t ; \quad \beta^{2}(t) \approx 6.566^{2}\left(0.9 e^{4.6 t}+0.1 e^{0.6 t}\right) ; \quad k \beta(0)=\sigma_{0}=7.249 \mathrm{GeV}^{-2} ; s_{0}=$ $1 G e V^{2}$; Furthermore, in SD $s^{\prime}=M^{2}, \quad e^{\Delta y}=\xi^{-1}=s / M^{2}$ while in DD: $s^{\prime}=s s_{0} /\left(M_{1}^{2} M_{2}^{2}\right), \quad s^{\prime}=$ $M_{1}^{2} M_{2}^{2} / s_{0}^{2}$.

The above formalism, based on the triple Regge limit (high missing masses), does not account for the low-mass resonances. In SD and DD they were included [3] by means of a dual-Regge model for the inelastic pPM vertex. We extend the above formalism to low-mass central systems by replacing the asymptotic expression of Pomeron-Pomeron total Pomeron-Pomeron cross section by its low missing mass extension [4] that includes also resonances.

Experimentally, central production in proton-proton collisions has been studied from the low energy range $\sqrt{s}=12.7-63 \mathrm{GeV}$ at the ISR at CERN up to the presently highest energy of $\sqrt{s}=13$ $\mathrm{TeV}$ available in Run II at the LHC. Ongoing data analysis of central production events include data taken by the COMPASS collaboration at the SPS [6], the CDF collaboration at the TEVATRON [7], the STAR collaboration at RHIC [8], and the ALICE and LHCb collaborations at the LHC [9, 10]. 


\section{Low missing masses, nuclear resonances. Basic formulae for single (SD), double (DD) and central (CED) diffraction}

- Total cross section: $p p \rightarrow X: \sigma^{t}(s)=g_{1}(0) g_{2}(0)\left(s / s_{0}\right)^{\alpha(0)-1}$; here $\beta_{1}=\beta_{2}, \alpha(t)$ is the Pomeron trajectory, whose form will be specified;

- Elastic scattering: $p p \rightarrow p p$,

$$
\frac{d \sigma}{d t}(s, t)=\frac{F(t)}{16 \pi}\left(s / s_{0}\right)^{2[\alpha(t)-1]},
$$

with the form factor $F(t)=g_{1}(t) g_{2}(t)$ to be specified;

- single diffraction dissociation, SD:

$$
16 \pi s \frac{d^{2} \sigma_{S D}}{d t d M^{2}}\left(s, t, M^{2}\right)=\left[g_{P p}(t)\left(\frac{M^{2}}{s}\right)^{1-2 \alpha(t)}\right]\left[\left(g_{P p p}(t) g_{P p}^{2}(0)\left(\frac{M^{2}}{s_{0}}\right)^{\alpha(0)-1}\right] .\right.
$$

With a simple, smooth Regge behaved total cross section for both $P p$ and $P P$ scattering one has:

$$
\sigma^{t}\left(M^{2}\right)=\left(M^{2}\right)^{0.1}+R\left(M^{2}\right)^{-0.5} .
$$

In what follows we will include also resonances in $M^{2}$.

Pomeron-Pomeron $(P P)$ scattering is similar to photon-photon scattering, the only main difference being in C-parities, respectively positive and negative, of the Pomeron and photon. High-virtuality $\gamma^{*} \gamma^{*}$ scattering is a favourite process in the framework of perturbative QCD, where the total cross section was calculated in Ref. [11]. In the leading-order BFKL

$$
\sigma_{t o t}^{\gamma^{*} \gamma^{*}}=\frac{\sigma_{0}}{\sqrt{Q_{1}^{2} Q_{2}^{2} Y}}\left(s / s_{0}\right)^{\lambda},
$$

where $Q_{i}^{2}=-q_{i}^{2}, \quad i=1,2$ is the photon virtuality and $Y=\ln \left(\frac{s}{s_{0}}\right)$. The quantity $\sigma_{0}$ is a free parameter and the exponent $\lambda \equiv \alpha_{P}^{B F K L}$ is the familiar BFKL eigenvalue $N_{c} \alpha_{s} 4 \ln 2 / \pi$. We recall that the transition from photon-photon to central Pomeron-Pomeron scattering is accompanied by the change of variables $t \rightarrow-Q^{2}$ and $s \rightarrow M^{2}$.

The one-by-one account of single resonances is possible, but not economic for the calculation of cross section, where a sequence of many resonances contributes at low masses. These resonances overlap and gradually disappear in the continuum at higher masses. An approach to account for many resonances, based on the idea of duality with a limited number of resonances lying on non-linear Regge trajectories, was suggested in Ref. [12]. Later on, this approach was used in Ref. [3] to calculate low mass single- and double-diffractive dissociation at the LHC.

For $s \rightarrow \infty$ and fixed $t$ it is Regge-behaved. Contrary to the Veneziano model, DAMA not only allows for, but rather requires the use of non-linear complex trajectories providing the resonance widths via the imaginary part of the trajectory.

$$
A\left(M_{X}^{2}, t\right)=a \sum_{i=f, P} \sum_{J} \frac{\left[f_{i}(t)\right]^{J+2}}{J-\alpha_{i}\left(M_{X}^{2}\right)} .
$$

The pole decomposition of the dual amplitude $A\left(M_{X}^{2}, t\right)$ is that of Eq. (8). The index $i$ sums over the trajectories which contribute to the amplitude. Within each trajectory, the second sum extends over the bound states of spin $J$. 
The pole residue $f(t)$ appearing in the $P P \rightarrow P P$ system is fixed by the dual model, in particular by the compatibility of its Regge asymptotics with Bjorken scaling and reads

$$
f(t)=\left(1-t / t_{0}\right)^{-2},
$$

where $t_{0}=0.71 \mathrm{GeV}^{2}$. Note that the residue enters with a power $(J+2)$ in Eq. (8), thereby strongly damping higher spin resonance contributions.

Following Ref. [3], we write the imaginary part of the amplitude $A\left(M_{X}^{2}, t\right)$, Eq. (8) as

$$
\mathfrak{J} m A\left(M_{X}^{2}, t\right)=a \sum_{i=f, P} \sum_{J} \frac{\left[f_{i}(t)\right]^{J+2} \mathfrak{J} m \alpha_{i}\left(M_{X}^{2}\right)}{\left(J-\operatorname{Re} \alpha_{i}\left(M_{X}^{2}\right)\right)^{2}+\left(\mathfrak{J} m \alpha_{i}\left(M_{X}^{2}\right)\right)^{2}},
$$

whence the total cross section is

$$
\sigma_{t}^{P P}\left(M_{X}^{2}\right)=\mathfrak{J} m A\left(M_{X}^{2}, t=0\right) .
$$

The Pomeron-Pomeron channel, $P P \rightarrow M_{X}^{2}$, couples to the Pomeron and $f$ channels. In order to calculate the $P P$ cross section, we therefore take into account the trajectories associated with the $\mathrm{f}_{0}(980)$ and the $\mathrm{f}_{2}(1270)$ resonances, and the Pomeron trajectory.

\section{Non-linear, complex Regge trajectories}

A non-trivial task for analytic models of Regge trajectories consists in deriving the imaginary part of the trajectory from the seemingly linearly increasing real part [13, 14]. A dispersion relation connects the real and imaginary part of the trajectory.

We follow Ref. [13] (see also [3, 4]) to relate the nearly linear real part of the meson trajectory to its imaginary part

$$
\mathfrak{R} e \alpha(s)=\alpha(0)+\frac{s}{\pi} P V \int_{0}^{\infty} d s^{\prime} \frac{\mathfrak{J} m \alpha\left(s^{\prime}\right)}{s^{\prime}\left(s^{\prime}-s\right)} .
$$

The imaginary part is related to the decay width by

$$
\Gamma\left(M_{R}\right)=\frac{\mathfrak{J} m \alpha\left(M_{R}^{2}\right)}{\alpha^{\prime} M_{R}} .
$$

The quantity $\alpha^{\prime}$ in Eq. (13) denotes the derivative of the real part, $\alpha^{\prime}=\frac{d \mathfrak{R} e \alpha(s)}{d s}$. The relation between $\Gamma(M)$ and $\mathfrak{I} m \alpha(\mathrm{s})$ requires $\mathfrak{I} m \alpha(s)>0$. In a simple analytical model, the imaginary part is chosen as a sum of single threshold terms [13]

$$
\mathfrak{J} m \alpha(s)=\sum_{n} c_{n}\left(s-s_{n}\right)^{1 / 2}\left(\frac{s-s_{n}}{s}\right)^{\left|\mathfrak{R} e \alpha\left(s_{n}\right)\right|} \theta\left(s-s_{n}\right)
$$

\section{$3.1 f$ mesons and their trajectories}

Apart from the Pomeron trajectory, to be discussed below, the direct-channel $f$ trajectories are essential in the PP channel. Guided by conservation of quantum numbers, we include two $f$ trajectories, the first labelled $f_{1}$ defined by the resonances $\mathrm{f}_{0}(980), \mathrm{f}_{1}(1420), \mathrm{f}_{2}(1810), \mathrm{f}_{4}(2300)$, and the second labelled $f_{2}$ defined by the resonances $\mathrm{f}_{2}(1270), \mathrm{f}_{4}(2050)$ and $\mathrm{f}_{6}(2510)$. The real and imaginary parts of these $f$ trajectories as well as the values of the parameters fitted to the spectroscopic data can be found in Ref. [4]. The $\mathrm{f}_{0}(500)$ resonance is taken as an isolated resonance with a Breit-Wigner parameterisation as described in Ref. [4]. 


\subsection{The Pomeron trajectory}

While ordinary meson trajectories can be fitted both in the resonance and scattering region corresponding to positive and negative values of the argument, the parameters of the Pomeron trajectory can only be determined in the scattering region $M^{2}<0$. A fit of the nonlinear Pomeron trajectory to high-energy $p p$ and $p \bar{p}$ data is discussed, e.g. in Ref. [5].

$$
\alpha_{P}\left(M^{2}\right)=1 .+\varepsilon+\alpha^{\prime} M^{2}-c \sqrt{s_{0}-M^{2}},
$$

with $\varepsilon=0.08, \alpha^{\prime}=0.25 \mathrm{GeV}^{-2}$ and $\mathrm{s}_{0}$ the two pion threshold $\mathrm{s}_{0}=4 \mathrm{~m}_{\pi}^{2}$. The value of $\mathrm{c}$ is set $\mathrm{c}=\alpha^{\prime} / 10=0.025$.

In order to be consistent with the mesonic trajectories shown above, the linear term in Eq. (15) is replaced by a heavy threshold mimicking linear behaviour in the mass region of interest $(\mathrm{M}<5 \mathrm{GeV})$,

$$
\alpha_{P}\left(M^{2}\right)=\alpha_{0}+\alpha_{1}\left(2 m_{\pi}-\sqrt{4 m_{\pi}^{2}-M^{2}}\right)+\alpha_{2}\left(\sqrt{M_{H}^{2}}-\sqrt{M_{H}^{2}-M^{2}}\right),
$$

where $M_{H}$ is an effective heavy threshold set at $M=3.5 \mathrm{GeV}$. The coefficients $\alpha_{0}, \alpha_{1}$ and $\alpha_{2}$ are chosen such that the Pomeron trajectory of Eq. (16) has a low energy behaviour as defined by Eq. (15).

The above trajectory is an oversimplified ansatz. An alternative to Eq. (15) is:

$$
\alpha_{P}\left(M^{2}\right)=\frac{1+\epsilon+\alpha^{\prime} M^{2}}{1-c \sqrt{s_{0}-M^{2}}} .
$$

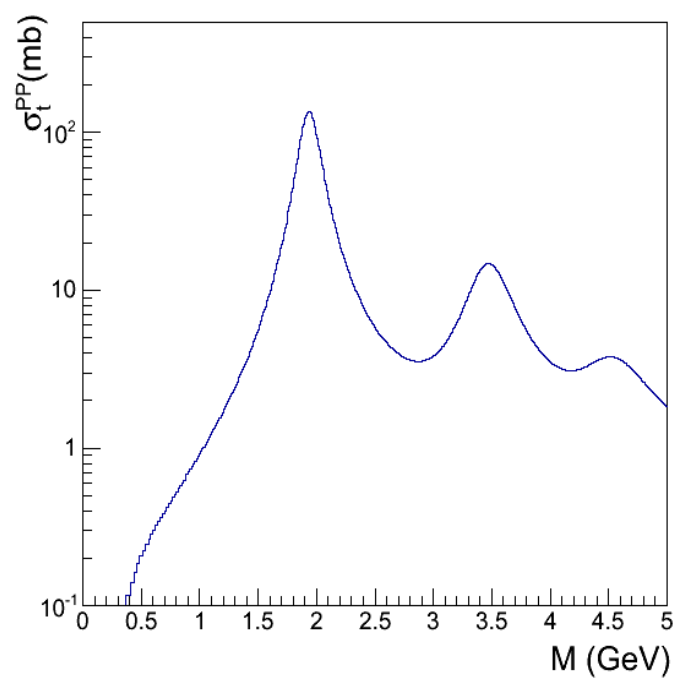

Figure 2. Direct-channel Pomeron contribution to PP total cross section.

Its contribution to the Pomeron-Pomeron total cross section is shown in Fig. 2. Contrary to Eq. (15), used in Ref. [4], it predicts glueballs whose widths increase with their masses, as one expects. 


\section{Pomeron-Pomeron total cross section}

The Pomeron-Pomeron cross section was calculated from the imaginary part of the amplitude by use of the optical theorem

$$
\sigma_{t}^{P P}\left(M^{2}\right)=\mathfrak{J} m A\left(M^{2}, t=0\right)=\sum_{i=f, P} \sum_{J} \frac{\left[f_{i}(0)\right]^{J+2} \mathfrak{J} m \alpha_{i}\left(M^{2}\right)}{\left(J-\mathfrak{R} e \alpha_{i}\left(M^{2}\right)\right)^{2}+\left(\mathfrak{J} m \alpha_{i}\left(M^{2}\right)\right)^{2}} .
$$

The index $i$ in Eq. (18) sums over the trajectories which contribute to the cross section, in our case the $f_{1}, f_{2}$ and the Pomeron trajectory discussed above. Within each trajectory, the summation extends over the resonances of spin $J$.

To summarize, a formalism to calculate central diffractive production of mesons is presented. Details on SD and DD can be found in Ref. [3], while those on meson production the collisions of Pomerons are in Ref. [4].

\section{References}

[1] K.A. Goulianos, Phys. Rep. 101(1983) 169.

[2] Robert Ciesielski, Konstantin Goulianos, MBR Monte Carlo Simulation in PYTHIAS, hep$\mathrm{ph} / 1205.1446$.

[3] L. Jenkovszky, O. Kuprash, J.W. Lamsa, V.K. Magas, and R. Orava, Phys.Rev.D83 (2011) 056014, arXiv:1011.0664.

[4] R. Fiore, L. Jenkovszky, and R. Schicker, Resonance production in Pomeron-Pomeron collisions at the $L H C$, to be published.

[5] L. Jenkovszky, Rivista Nuovo Cim., 10 N12, (1987), p.1.

[6] A. Austregesilo, for the COMPASS Collab., Proceedings 15th Conference on Elastic and Diffractive Scattering, Saariselka, Sept 2013, arXiv:1310.3190.

[7] M. Albrow, for the CDF Collab., Int. J. Mod.Phys. A29 (2014) 28, 1446009, arXiv:1409.0462.

[8] L. Adamczyk, W. Guryn and J. Turnau, Int. J. Mod.Phys. A29 (2014) 28, 1446010, arXiv:1410.5752.

[9] R. Schicker, Int. J. Mod. Phys. A29 (2014) 28, 1446015, arXiv:1411.1283.

[10] R. McNulty, Int. J. Mod.Phys. A29 (2014) 28, 1446003, arXiv:1409.8113. arXiv:1402.2170.

[11] J. Bartels, A. De Roeck, and H. Lotter, Phys. Lett. B389 (1996) 742; F. Caporale, D.Y. Ivanov, A. Papa, Eur. Phys. J. C 58 (2008) 1.

[12] R. Fiore, A. Flachi, L.L. Jenkovszky, A. Lengyel and V. Magas, Phys. Rev. D69 (2004), 014004, hep-ph/0308178.

[13] R. Fiore, L.L. Jenkovszky, V. Magas, F. Paccanoni, A. Papa, Eur.Phys.J. A10 (2001) 217-221, hep-ph/0011035.

[14] R.Fiore, L.L. Jenkovszky, F. Paccanoni, A. Prokudin, Phys.Rev.D70 (2004)054003, hep$\mathrm{ph} / 0404021$.

[15] R. Fiore, L.L. Jenkovszky, V. Magas, F. Paccanoni and A. Papa, Phys. Part. Nucl.31 (2000) 46, hep-ph/9911503. 